\title{
DIFERENCIAÇÃO EMOCIONAL E REGULAÇÃO EMOCIONAL EM ADULTOS PORTUGUESES
}

Filipa Machado $\mathrm{Vaz}^{1}$

Carla Martins ${ }^{2}$

Eva Costa Martins ${ }^{3}$

Resumo: Este estudo empírico pretendeu caracterizar o repertório e capacidade de diferenciação emocional e estratégias de regulação emocional em adultos, como estas se relacionam, bem como avaliar como variam em função de características sócio-demográficas. Uma amostra de 824 indivíduos entre os 18 e os 65 anos respondeu a dois questionários, a Escala de Avaliação do Repertório e Capacidade de Diferenciação Emocional e o Questionário de Regulação Emocional. Os resultados evidenciaram que, quanto maior é o repertório e a diferenciação emocional, menor é a utilização da supressão emocional. Adicionalmente, uma maior capacidade de diferenciação emocional está associada a uma maior reavaliação cognitiva. Apesar de não haver diferenças de género em termos de repertório e diferenciação emocional, os homens recorrem mais à supressão emocional. Indivíduos com o Ensino Básico referem menor repertório emocional do que indivíduos com um nível de escolaridade mais elevado (Secundário ou Superior/Pós-Graduado) e empregam mais ambas as estratégias de regulação emocional.

Palavras-chave: Emoções, diferenciação emocional, regulação emocional, idade adulta.

Emotional differentiation and emotion regulation in Portuguese adults (Abstract): This empirical study as well as aimed at characterizing adults' emotional repertoire and differentiation, as well as emotional regulation strategies, and assessing how these vary as a function of socio-demographic characteristics. It also focused on the relationship between emotional repertoire and differentiation and emotional regulation. A sample of 824 adults aged 18 to 65 years answered two self-reports, the Range and Differentiation of Emotional Experience Scale and the Emotion Regulation Questionnaire. Results showed that higher emotional repertoire and differentiation is associated with lower emotional suppression. Additionally,

\footnotetext{
${ }^{1}$ Escola de Psicologia, Universidade do Minho.

2 Escola de Psicologia, Universidade do Minho.

3 ISMAI, Instituto Superior da Maia.

Contacto para correspondência: cmartins@iep.uminho.pt
} 
higher emotional differentiation is coupled wih higher cognitive reappraisal. No gender differences were found in terms of emotional repertoire and differentiation, but males use more emotional suppression. Finally, individuals with lower educational lever report lower emotional repertoire than individuals with higher educational level and use more both emotional regulation strategies.

Keywords: Emotions, emotional differenciation, emotion regulation, adulthood.

\section{Introdução}

As emoções, enquanto reacções subjectivas e idiossincráticas a um determinado evento do ambiente interno ou externo, são caracterizadas por mudanças fisiológicas, cognitivas, experienciais e comportamentais, que permitem ao indivíduo a atribuição de um significado à experiência e o preparam para a acção (Kring \& Bachorowski, 1999; Sroufe, 1996). Assim, as emoções não se resumem à reacção fisiológica do organismo a um estímulo: para experienciar uma emoção, o indivíduo tem de atribuir um significado a essa activação, em função do tipo de activação fisiológica experienciada, do contexto em que ocorre, das experiências passadas e das memórias episódicas construídas (Barrett, 2006). Este processo denomina-se diferenciação emocional a qual, em articulação com a activação emocional, conduz o indivíduo a experienciar uma emoção. Na sequência deste processo de diferenciação emocional, surgem determinadas estratégias de regulação emocional que o indivíduo utiliza para expressar ou não as suas emoções (Barrett, 2006).

\section{Diferenciação emocional na idade adulta}

A diferenciação emocional consiste num processo de representação mental da emoção, de simbolização e consequente expansão da reacção fisiológica experienciada, sendo constituída por duas dimensões: (i) o repertório de experiências emocionais, relacionado com a diversidade de emoções experienciadas por cada indivíduo e (ii) a capacidade para realizar distinções subtis entre emoções similares (Kang \& Shaver, 2004). Se alguns indivíduos experienciam as emoções de uma forma altamente diferenciada, distinguindo-as minuciosamente de entre uma ampla variedade de emoções possíveis, outros vivenciam-nas de uma forma relativamente indiferenciada, utilizando termos emocionais sem grande distinção (Barrett, 2004; Lindquist, Barrett, Bliss-Moreau \& Russell, 2006). De facto, indivíduos com boa capacidade de diferenciação emocional, são capazes de saber o que estão a sentir e porquê, e têm acesso a um nível maior de conhecimento emocional 
(Barrett \& Russel, 1998). Em contraste, indivíduos com reduzida capacidade de diferenciação emocional utilizam categorias supra-ordenadas como forma de categorização das suas experiências (por exemplo, categorizando como desagradáveis, emoções que poderiam ser classificadas como tristeza, raiva ou medo), experienciando as suas emoções de forma pouco específica (Lindquist et al., 2006). Consequentemente, a capacidade de diferenciação emocional assume um papel de relevo na forma como o indivíduo regula as suas emoções (Barrett, 1998).

\section{Regulação emocional na idade adulta}

A regulação emocional traduz-se num conjunto de estratégias que o indivíduo utiliza para aumentar, manter ou diminuir uma ou mais componentes de uma determinada resposta emocional, podendo ocorrer em todas as dimensões do processamento emocional, nomeadamente a nível fisiológico, cognitivo, comportamental, experiencial e social (Gross, 1999). A nível fisiológico, o processo de regulação emocional permite que a activação emocional seja redireccionada, controlada, modelada e modificada, de modo a permitir ao indivíduo funcionar de forma adaptativa em situações emocionalmente activadoras (Cicchetti, Ackerman, \& Izard, 1995). A nível cognitivo, o indivíduo revê a situação e modifica o significado das suas emoções, de modo a transformar ou regular as suas reacções emocionais. A nível comportamental, os indivíduos podem, por um lado, controlar o que pretendem expressar e o que pretendem suprimir e, por outro, gerir as situações às quais decidem expôr-se, o que, por sua vez, conduz à procura ou evitamento de determinado estímulo que evoque as emoções (Greenberg, 2002).

A investigação acerca das diferenças individuais ao nivel da regulação emocional no adulto tem-se centrado principalmente no estudo de duas estratégias de regulação emocional temporalmente distintas no processo emocional: reavaliação cognitiva (estratégia de regulação emocional antecedente à resposta emocional) e supressão emocional (estratégia de regulação emocional centrada na resposta emocional) (Gross \& Thompson, 2007). A reavaliação cognitiva permite a modificação da totalidade da sequência emocional, possibilitando a experiência de mais emoções positivas e de menos emoções negativas, sem consequências fisiológicas, cognitivas ou interpessoais desadaptativas (Gross \& John, 2003). A supressão emocional cinge-se à modificação da componente comportamental e expressão emocional, não reduzindo a experiência emocional negativa, estando associada a uma diminuição de experiências emocionais positivas, a dificuldades na elaboração de memórias relacionadas com o contexto social, comprometendo o funcionamento social a vários níveis (Srivastava, McGonigal, Tamir, John, \& Gross, 2009). 


\section{Diferenciação emocional e regulação emocional na idade adulta}

Estudos recentes sublinham a necessidade de aprofundar a relação entre a capacidade de diferenciação emocional e a eficácia das estratégias de regulação emocional utilizadas (Barrett, Gross, Conner, \& Benvenuto, 2001; Wranik, Barrett, \& Salovey, 2007). Sabermos com exactidão o que estamos a sentir permite o acesso, não só a informação sobre a significância da situação, mas também a estratégias de coping a utilizar. Por outras palavras, se uma maior capacidade de diferenciação emocional está relacionada com uma maior activação do conhecimento relativo às emoções, esta, por sua vez, poderá estar associada à capacidade para regular as emoções (Wranik, Barrett, \& Salovey, 2007). Nesta linha, vários estudos demonstram que os indivíduos com uma maior capacidade de diferenciação emocional apresentam uma maior diversidade e adequação de estratégias de regulação emocional (Barrett et al., 2001; Philippot, Baeyens, Douilliez, \& Francart, 2004; Tugade, Barrett, \& Gross, 2004). Assim, a capacidade de diferenciação e regulação emocional parecem estar positivamente correlacionadas, na medida em que o conhecimento emocional poderá estar na base da avaliação da acção e da percepção, permitindo ao indivíduo decidir quando e como regular as suas emoções de uma forma adaptativa (Barrett, 1998).

O presente estudo tem um duplo objectivo. Em primeiro lugar, pretende-se caracterizar o repertório e a capacidade de diferenciação emocional e estratégias de regulação emocional, numa amostra de adultos, e analisar as suas intercorrelações. Em segundo lugar, pretende-se analisar como o repertório e a capacidade de diferenciação emocional e de regulação emocional variam em função de características sócio-demográficas dos indivíduos, nomeadamente, idade, género e nível de escolaridade.

\section{Método}

\section{Participantes}

A amostra é constituída por 824 indivíduos com idades compreendidas entre os 18 e os 65 anos $(M=27,37, D P=7,99)$. A maioria dos indivíduos é do sexo masculino $(N=457,55,5 \%)$, completou o Ensino Secundário $(N=578,70,1 \%)$ e é solteiro $(N=569,69,1 \%)$. (Tabela 1$)$ 
Tabela 1. Descrição da amostra $(N=824)$

\begin{tabular}{llll}
\hline & & $\boldsymbol{N}$ & $\mathbf{( \% )}$ \\
\hline Sexo & Masculino & 457 & $(55,5)$ \\
& Feminino & 367 & $(44,5)$ \\
\hline Escolaridade & Ensino Básico & 141 & $(17,1)$ \\
& Ensino Secundário & 578 & $(70,1)$ \\
& Ensino Superior ou Pós Graduado & 104 & $(12,6)$ \\
\hline \multirow{2}{*}{ Estado Civil } & Solteiro & 569 & $(69,1)$ \\
& Casado/União de Facto & 219 & $(26,6)$ \\
& Divorciado ou Viúvo & 36 & $(4,4)$ \\
\hline
\end{tabular}

\section{Medidas}

Para este estudo foram utilizados dois instrumentos de avaliação previamente adaptados para a população adulta portuguesa: a Escala de Avaliação do Repertório e Capacidade de Diferenciação Emocional (Range and Differentiation of Emotional Experience Scale, Kang \& Shaver, 2004; versão portuguesa de Machado Vaz, Martins, \& Vieira) e o Questionário de Regulação Emocional (Emotion Regulation Questionnaire, Gross \& John, 2003; versão portuguesa de Machado Vaz, Martins, \& Vieira).

A Escala de Avaliação do Repertório e Capacidade de Diferenciação Emocional é uma escala de auto-relato constituída por catorze itens, avaliados numa escala de Likert de 7 pontos (1 "Não me identifico" até 7 "Identifico-me totalmente") que se distribuem por duas sub-escalas: Repertório Emocional e Diferenciação Emocional. A sub-escala Repertório Emocional $\left(\alpha_{\text {Cronbach }}=0,63\right.$ na versão portuguesa $)$ é constituída por seis itens que avaliam a variedade ou repertório de diferentes emoções experienciadas pelo indivíduo (ex., "Experiencio uma grande variedade de emoções"). Os valores mais elevados indicam que o indivíduo possui um maior repertório emocional. A sub-escala Diferenciação Emocional $\left(\alpha_{\text {Cronbach }}=0,82\right)$ é constituída por oito itens que avaliam a capacidade do indivíduo para fazer distinções subtis entre emoções similares (ex., "Sou bom a distinguir diferenças subtis no significado de palavras emocionais muito relacionadas"). Os valores mais elevados indicam uma maior capacidade para diferenciar categorias emocionais similares.

O Questionário de Regulação Emocional é uma medida de auto-relato constituída por dez itens, avaliados numa escala de Likert de 7 pontos (1 "Discordo totalmente" até 7 "Concordo totalmente"). Este instrumento é constituído por duas sub-escalas: a) Reavaliação Cognitiva $\left(\alpha_{\text {Cron- }}\right.$ bach $=0,76$ ), que integra seis itens (ex., "Eu controlo as minhas emoções 
modificando a forma de pensar acerca da situação em que me encontro"), e b) Supressão Emocional $\left(\alpha_{\text {Cronbach }}=0,65\right)$, que integra quatro itens (ex., "Quando estou a experienciar emoções negativas, faço tudo para não as expressar"). Os resultados elevados em cada uma das sub-escalas reflectem uma maior utilização da estratégia de regulação emocional específica.

\section{Procedimento}

O estudo foi implementado junto de uma amostra de adultos em doze instituições de ensino superior, instituições de ensino profissional e centros de reconhecimento e validação de competências em várias zonas de Portugal Continental.

No momento da recolha de dados os indivíduos foram informados, verbalmente e por escrito, acerca dos objectivos do estudo, tendo sido garantida a confidencialidade de todos os dados recolhidos. A administração dos instrumentos foi colectiva. Os indivíduos preencheram também um pequeno questionário sócio-demográfico com questões sobre o sexo, idade, estado civil e nível de escolaridade.

\section{Resultados}

Repertório e Capacidade de Diferenciação Emocional e Regulação Emocional

A Tabela 2 apresenta as medidas descritivas das dimensões avaliadas - repertório emocional e diferenciação emocional bem como as duas estratégias de regulação emocional - reavaliação cognitiva e supressão emocional. Todas as dimensões, à excepção da Supressão Emocional, têm uma média acima do ponto médio da escala.

Em termos de intercorrelações, apresentadas na Tabela 3, foram encontradas correlações positivas significativas entre as dimensões do mesmo instrumento (repertório emocional e diferenciação emocional, $r=0,37, p<0,001$; reavaliação cognitiva e supressão emocional, $r=0,30$, $p<0,001)$. Para além disso, quanto maior o repertório emocional dos indivíduos, menor a utilização da supressão emocional como estratégia de regulação emocional $(r=-0,26, p<0,001)$. Por outro lado, indivíduos que expressam maior capacidade de diferenciação emocional, tendem a recorrer mais à reavaliação cognitiva $(r=0,18, p<0,001)$ e menos à supressão emocional $(r=-0,07, p<0,05)$ enquanto estratégias de regulação emocional. 
Tabela 2. Medidas descritivas das variáveis avaliadas $(N=824)$

\begin{tabular}{|c|c|c|c|}
\hline & & Média (DP) & Min-Máx \\
\hline \multirow{2}{*}{$\begin{array}{l}\text { Repertório e Capaci- } \\
\text { dade de Diferenciação } \\
\text { Emocional }\end{array}$} & Repertório Emocional & $4,83(0,85)$ & $2,67-7,00$ \\
\hline & $\begin{array}{l}\text { Diferenciação Emocio- } \\
\text { nal }\end{array}$ & $4,54(0,92)$ & $1,75-7,00$ \\
\hline \multirow{2}{*}{ Regulação Emocional } & Reavaliação Cognitiva & $4,36(1,17)$ & $1,00-7,00$ \\
\hline & Supressão Emocional & $3,45(1,24)$ & $1,00-7,00$ \\
\hline
\end{tabular}

Tabela 3. Intercorrelações entre Repertório Emocional, Diferenciação Emocional, Reavaliação Cognitiva e Supressão Emocional e Idade $(N=824)$

\begin{tabular}{|c|c|c|c|c|}
\hline & $\begin{array}{l}\text { Repertório } \\
\text { emocional }\end{array}$ & $\begin{array}{c}\text { Diferenciação } \\
\text { Emocional }\end{array}$ & $\begin{array}{c}\text { Reavaliação } \\
\text { Cognitiva }\end{array}$ & $\begin{array}{l}\text { Supressão } \\
\text { Emocional }\end{array}$ \\
\hline Repertório emocional & - & & & \\
\hline Diferenciação Emocional & $0,37 * * *$ & - & & \\
\hline Reavaliação Cognitiva & $-0,05$ & $0,18 * * *$ & - & \\
\hline Supressão Emocional & $-0,26 * * *$ & $-0,07^{*}$ & $0,30 * * *$ & - \\
\hline Idade & $-0,07^{*}$ & 0,05 & $0,12 * * *$ & $0,14 * * *$ \\
\hline
\end{tabular}

Nota: $* * * p<0,001 ; * p<0,05$

A idade dos indivíduos correlaciona-se negativamente com o repertório emocional $(r=-0,07, p<0,05)$ e positivamente com a reavaliação cognitiva $(r=0,12, p<0,001)$ e a supressão emocional $(r=0,14, p<0,001)$. Assim, indivíduos mais velhos relatam menor repertório emocional e maior utilização de ambas as estratégias de regulação emocional.

\section{Diferenças de género e nível de escolaridade}

A Tabela 4 apresenta os resultados relativos às MANOVAs utilizadas para investigar diferenças de género ao nível das dimensões emocionais avaliadas.

No que toca ao repertório e diferenciação emocional, não foram encontradas diferenças significativas entre homens e mulheres (Wilks' Lambda $=0,99, F(2,821)=1,34$, n.s. $)$.

Em contraste, foram encontradas diferenças multivariadas entre os grupos no que diz respeito à utilização de estratégias de regulação emocional (Wilks' Lambda $=0,98, F(2,821)=7,14, p<0,001)$. Testes univariados revelaram que os homens recorrem mais à supressão emocional como estra- 
tégia de regulação emocional do que as mulheres. Não foram encontradas diferenças de género em termos de reavaliação cognitiva.

Tabela 4. Diferenças de género ao nível do Repertório Emocional, Diferenciação Emocional, Reavaliação Cognitiva e Supressão Emocional.

\begin{tabular}{lccccc}
\hline & \multicolumn{2}{c}{$\begin{array}{c}\text { Masculino } \\
(\boldsymbol{N = 4 5 7 )}\end{array}$} & \multicolumn{2}{c}{$\begin{array}{c}\text { Feminino } \\
(\boldsymbol{N = 3 6 7 )}\end{array}$} & \\
\cline { 2 - 5 } & Média & $(D P)$ & Média & $(D P)$ & $F(1,822)$ \\
\hline Repertório Emocional & 4,78 & $(0,87)$ & 4,88 & $(0,83)$ & 2,49 \\
$\begin{array}{l}\text { Diferenciação Emocio- } \\
\text { nal }\end{array}$ & 4,54 & $(0,92)$ & 4,55 & $(0,92)$ & 0,032 \\
$\begin{array}{l}\text { Reavaliação Cognitiva } \\
\text { Supressão Emocional }\end{array}$ & 4,32 & $(1,18)$ & 4,41 & $(1,16)$ & 1,25 \\
\hline
\end{tabular}

Nota: $* * p<0,01$

A Tabela 5 apresenta os resultados relativos às MANOVAs para avaliação do efeito do nível de escolaridade nas dimensões avaliadas.

Tabela 5. Diferenças do nível de escolaridade ao nível do Repertório Emocional, Diferenciação Emocional, Reavaliação Cognitiva e Supressão Emocional.

\begin{tabular}{lccccccc}
\hline & \multicolumn{2}{c}{$\begin{array}{c}\text { Básico } \\
(\boldsymbol{N = 1 4 1 )}\end{array}$} & \multicolumn{2}{c}{$\begin{array}{c}\text { Secundário } \\
(\boldsymbol{N}=\mathbf{5 7 8})\end{array}$} & \multicolumn{2}{c}{$\begin{array}{c}\text { Superior } \\
\text { Pós-Graduado } \\
(\boldsymbol{N}=\mathbf{1 0 4})\end{array}$} & \\
\cline { 2 - 6 } & Média & $(\mathrm{DP})$ & Média & $(\mathrm{DP})$ & Média & $(\mathrm{DP})$ & $F(2,820)$ \\
\hline $\begin{array}{l}\text { Repertório } \\
\text { Emocional }\end{array}$ & 4,49 & $(0,62)$ & 4,91 & $(0,88)$ & 4,83 & $(0,90)$ & $14,31^{* * *}$ \\
$\begin{array}{l}\text { Diferenciação } \\
\text { Emocional }\end{array}$ & 4,47 & $(0,95)$ & 4,58 & $(0,90)$ & 4,45 & $(0,99)$ & 1,36 \\
$\begin{array}{l}\text { Reavaliação } \\
\text { Cognitiva }\end{array}$ & 4,81 & $(1,28)$ & 4,27 & $(1,11)$ & 4,20 & $(1,18)$ & $13,64^{* * *}$ \\
$\begin{array}{l}\text { Supressão } \\
\text { Emocional }\end{array}$ & 3,89 & $(1,32)$ & 3,39 & $(1,18)$ & 3,16 & $(1,28)$ & $12,92^{* * *}$ \\
\hline
\end{tabular}

Nota: $* * * p<0,001$ 
Foram encontradas diferenças multivariadas entre os indivíduos com diferentes níveis de escolaridade em termos do seu repertório e diferenciação emocional (Wilks' Lambda $=0,96, F(4,1638)=7,63, p<0,001$ ). Testes univariados evidenciaram diferenças ao nível do repertório emocional, mas não em termos de diferenciação emocional. Testes post-hoc de Scheffé revelaram que os indivíduos com o Ensino Básico referem menor Repertório Emocional do que os indivíduos do Ensino Secundário ou Superior, os quais não se diferenciam entre si.

Por último, pretendemos avaliar a influência do nível de escolaridade na implementação de estratégias de regulação emocional. Uma vez mais, foram encontradas diferenças multivariadas ao nível das estratégias de regulação emocional em função do nível de escolaridade dos participantes (Wilks' Lambda $=0,95, F(4,1638)=10,38, p<0,001)$. Testes univariados evidenciaram diferenças ao nível de ambas as estratégias de regulação emocional. Tanto ao nível da utilização da reavaliação cognitiva como da supressão emocional, testes post-hoc de Scheffé revelaram que os indivíduos com o Ensino Básico utilizam mais ambas as estratégias de regulação emocional do que os indivíduos com nível de escolaridade mais elevado (Secundário ou Superior/Pós-Graduado).

\section{Discussão}

Este estudo empírico teve o duplo objectivo de caracterizar o repertório e a capacidade de diferenciação emocional e de regulação emocional e suas interrelações, bem como avaliar como estas variam em função de características sócio-demográficas.

Nesta amostra de adultos portugueses foi encontrada uma correlação positiva entre o repertório emocional e a diferenciação emocional, resultados consistentes com a evidência empírica existente (cf. Kang \& Shaver, 2004). Assim, quanto maior for o repertório emocional do indivíduo, maior será a sua capacidade de diferenciar as suas emoções, podendo estas duas dimensões caracterizar a complexidade emocional de uma pessoa (Kang \& Shaver, 2004). Também entre as duas estratégias de regulação emocional foi detectada uma correlação positiva. Este resultado é surpreendente na medida em que reavaliação cognitiva e supressão emocional são, de alguma forma, estratégias de regulação emocional diversas e cuja utilização se esperaria independente (Gross \& John, 2003). De facto, enquanto a reavaliação cognitiva é uma estratégia de regulação emocional adaptativa, na medida em que permite a experiência de mais emoções positivas e de menos emoções negativas, já a supressão emocional poderá ser considerada desadaptativa, dado que não reduz a experiência emocional negativa experienciada e 
está associada a vários efeitos negativos no funcionamento social (Srivastava et al., 2009).

A supressão emocional revelou uma correlação negativa com o repertório e a diferenciação emocional. Indivíduos que expressam maior repertório e capacidade de diferenciação emocional tendem a recorrer menos à supressão emocional enquanto estratégia de regulação emocional. Por outro lado, indivíduos que expressam maior capacidade de diferenciação emocional tendem a recorrer mais à reavaliação cognitiva e esta não está associada ao repertório emocional. Existe um amplo consenso de que um conhecimento elaborado acerca das emoções está relacionado com uma melhor regulação emocional em adultos (Wranik, Barrett, \& Salovey, 2007), podendo igualmente, conjecturar-se esta relação a respeito de uma maior variabilidade emocional. Mas este pode não ser o caso. Kang e Shaver (2004) referem que indivíduos com maior diferenciação emocional tendem a perceber as emoções que experienciam com maior clareza, enquanto as pessoas com uma propensão para experienciar uma grande amplitude de emoções têm propensão para terem sentimentos fortes que podem ser desorganizadores. Assim, mais importante do que o indivíduo ter um elevado número de experiências emocionais, é este ser capaz de diferenciar as emoções, compreender a sua função e reflectir sobre as mesmas. Tais competências, por seu turno, revelam-se fundamentais para uma boa regulação emocional, permitindo aos indivíduos decidir o que querem expressar ou suprimir (Barrett et al., 2001). Os nossos resultados parecem, pois, apoiar a ideia de que indivíduos com uma maior capacidade de diferenciação serão capazes de regular as suas emoções de forma mais adaptativa (recorrendo menos à supressão emocional e mais à reavaliação cognitiva) do que indivíduos com dificuldades de diferenciação emocional (Barrett, 1998; Barrett et al., 2001). O repertório emocional apresenta-se como uma dimensão menos diferenciadora das duas estratégias, visto somente se correlacionar com a supressão emocional. Este resultado poderá reforçar a investigação que mostra a ligação entre a estratégia regulatória de supressão e nomeadamente uma diminuição de experiências emocionais positivas (John \& Gross, 2004).

No entanto, é necessário apontar o facto de todos os nossos coeficientes de correlação, embora significativos, serem de baixa magnitude. Desta forma, apesar de podermos concluir que há associações significativas entre as dimensões estudadas, algumas delas (ex., maior diferenciação emocional, menor supressão emocional) poderão ser devido à dimensão da amostra e não a relações que, de facto, existam na população adulta portuguesa.

Foram encontradas correlações entre a idade e os constructos emocionais estudados, resultados consistentes com outras evidências empíricas: negativas com o repertório emocional e positivas com ambas as estratégias emocionais. Por outras palavras, indivíduos mais velhos relatam menor reper- 
tório emocional (Lane, Sechrest \& Riedel, 1998) e maior utilização de ambas as estratégias de regulação emocional. No entanto, uma vez mais, as correlações são de baixa magnitude pelo que não devem ser sobrevalorizadas.

Em termos da relação entre as dimensões emocionais avaliadas e o género, embora não tenham sido encontradas diferenças no que toca ao repertório e diferenciação emocional, tal como no estudo original do questionário, os homens relatam recorrer mais à supressão emocional como estratégia de regulação emocional do que as mulheres (Gross e John, 2003). De facto, nas sociedades ocidentais, expressar emoções é visto como sendo característico das mulheres, pelo que a supressão emocional por parte dos homens é compreensível (Brody, 2000).

O nosso estudo evidenciou, ainda, um efeito do nível de escolaridade. Concretamente, os indivíduos com o Ensino Básico reportam menor repertório emocional, associado a uma maior utilização de ambas as estratégias de regulação emocional do que indivíduos com um nível de escolaridade mais elevado (Secundário ou Superior/Pós-Graduado), os quais não diferem entre si. Estes resultados vão, de alguma forma, na mesma linha do resultado empírico de Lane e colaboradores que encontraram que baixa emotional awareness está associada a menos anos de escolaridade (Lane et al., 1998). Igualmente, estando a percepção da variabilidade emocional intimamente relacionada com as oportunidades educacionais e desenvolvimento da linguagem (Wranik, Barrett, \& Salovey, 2007), o nível de escolaridade poderá ser um indicador destes factores. A maior utilização de supressão emocional por parte dos indivíduos menos escolarizados é coerente com o seu menor repertório emocional. Como verificámos anteriormente, a estratégia de reavaliação cognitiva está associada à de supressão emocional, contrariamente a outros estudos, podendo justificar esta maior preponderância nos indivíduos com o Ensino Básico. Esta diferença característica ou não à população portuguesa necessita de mais investigação. É curioso verificar que, analisando os estudos com o instrumento, nos EUA e na Europa, as variáveis relacionadas com o NSE não são analisadas, talvez porque as amostras são constituídas maioritariamente por alunos universitários (cf. Gross \& John, 2003; Verduyn, Delvaux, Van Coillie, Tuerlinckx, \& Van Mechelen, 2009). Esta poderá ser uma importante linha de investigação futura.

Por fim, salientamos a importância de alargar este estudo a populações clínicas em contexto português, com especial relevo para perturbações em que as dificuldades em regular as emoções assumem relevância primordial, como sejam, por exemplo, as perturbações de humor e ansiedade (Campbell-Sills \& Barlow, 2007) ou a dependência do álcool (Sher \& Grekin, 2007), entre outros. 


\section{Referências}

Barrett, L. F. (1998). Discrete emotions or dimensions? The role of valence focus and arousal focus. Cognition and Emotion, 12, 579-599.

Barrett, L. F. (2004). Feelings or words? Understanding the content in self-report ratings of experienced emotion. Journal of Personality and Social Psychology, $87(2), 266-281$

Barrett, L. F. (2006). Solving the emotion paradox: Categorization and the experience of emotion. Personality and Social Psychology Review, 10, 20-46.

Barrett, L. F., Gross, J., Conner, T., \& Benvenuto, M. (2001). Emotion differentiation and regulation. Cognition and Emotion, 15, 713-724.

Barrett, L. F., \& Russell, J. A. (1998). Independence and bipolarity in the structure of current affect. Journal of Personality and Social Psychology, 74, 967-984.

Brody, L. R. (2000). The socialization of gender differences in emotional expression: Display rules, infant temperament, and differentiation. In A. H. Fischer (Ed.), Gender and emotion: Social psychological perspectives (pp. 24-47). New York: Cambridge University Press.

Campbell-Sills, L., \& Barlow, D. H. (2007). Incorporating emotion regulation into conceptualizations and treatments of anxiety and mood disorders. In J. J. Gross (Ed.), Handbook of emotion regulation (pp. 542-559). New York: Guilford.

Cicchetti, D., Ackerman, B., \& Izard, C. (1995). Emotions and emotion regulation in developmental psychopathology. Development and Psychopathology, 7, 1-10.

Greenberg, L. S. (2002). Emotion-focused therapy: coaching clients to work through their feelings. Washington DC: American Psychological Association.

Gross, J. J. (1999). Antecedent and response focused emotion regulation: Journal of Personality and Social Psychology, 74(1), 224-237.

Gross, J. J., \& John, O. P. (2003). Individual differences in two emotion regulation processes. Journal of Personality and Social Psychology, 85(2), 348-362.

Gross, J. J., \& Thompson, R. A. (2007). Emotion regulation: Conceptual foundations. In J. J. Gross (Ed.), Handbook of emotion regulation (pp. 3-24). New York: Guilford.

John, O. P., \& Gross, J. J. (2004). Healthy and unhealthy emotion regulation: Personality processes, individual differences, and lifespan development. Journal of Personality, 72, 1301-1334.

Kang, S., \& Shaver, P. (2004). Individual differences in emotional complexity: their psychological implications. Journal of Personality, 72(4), 687-726.

Kring, A. M., \& Bachorowski, J. A. (1999). Emotions and psychopathology. Cognition and emotion, 13(5), 575-599.

Lane, R. D., Sechrest, L., \& Riedel, R. (1998). Sociodemographic correlates of alexithymia. Comprehensive Psychiatry, 39, 377-385.

Lindquist, K. A., Barrett, L. F., Bliss-Moreau, E., \& Russell, J. A. (2006). Language and perception of emotion. Emotion, 6(1), 125-138. 
Machado Vaz, F., Martins, C., \& Vieira, D. (Submetido). Psychometric properties of the Portuguese Version of the Emotion Regulation Questionnaire (ERQ). The Spanish Journal of Psychology.

Machado Vaz, F., Martins, C., \& Vieira, D. (Em preparação). The Portuguese Version of the Range and Differentiation of Emotional Experience Scale.

Philippot, P., Baeyens, C., Douilliez, C., \& Francart, B. (2004). Cognitive regulation of emotion: Application to clinical disorders. In P. Philippot \& R. S. Feldman (Eds.), The regulation of emotion (pp. 71-97). Mahwah, NJ: Erlbaum.

Sher, K. J., \& Grekin, E. R. (2007). Alcohol and affect regulation. In J. J. Gross (Ed.), Handbook of emotion regulation (pp. 560-580). New York: Guilford.

Srivastava, S., Tamir, M., McGonigal, K. M., John, O. P., \& Gross, J. J. (2009). The social costs of emotional suppression: A prospective study of the transition to college. Journal of Personality and Social Psychology, 96, 883-897.

Sroufe, A. (1996). Emotional Development. The organization of emotional life in the early years. New York: Cambridge University Press.

Tugade, M. M., Barrett, L. F., \& Gross, J. J. (2004, July). Matters of feeling precisely: Examining emotional granularity and emotion regulation in context. In S. J. Catanzaro (Chair) Individual differences in mood experience: Recent developments and future trends. Symposium at the annual meeting of the American Psychological Society, Chicago, IL.

Verduyn, P., Delvaux, E., Van Coillie, H., Tuerlinckx, F., \& Van Mechelen, I. (2009). Predicting the duration of emotional experience: Two experience sampling studies. Emotion, 9, 83-91.

Wranik, T., Feldman Barrett, L., \& Salovey, P. (2007). Intelligent emotion regulation: Is knowledge power? In J. Gross (Ed.), Handbook of emotion regulation (pp. 393-407). New York: Guilford. 